\title{
Genomic variation in the hybrids of white crucian carp and red crucian carp: evidence from ribosomal DNA
}

\author{
WANG Jing $^{\dagger}$, XIAO Jun ${ }^{\dagger}$, ZENG Ming ${ }^{\dagger}$, XU Kang, TAO Min, ZHANG Chun, DUAN Wei, \\ LIU WenBin, LUO KaiKun, LIU Yun \& LIU ShaoJun* \\ Key Laboratory of Protein Chemistry and Fish Developmental Biology of Ministry of Education of China, College of Life Sciences, Hunan \\ Normal University, Changsha 410081, China
}

Received August 8, 2014; accepted December 13, 2014; published online March 23, 2015

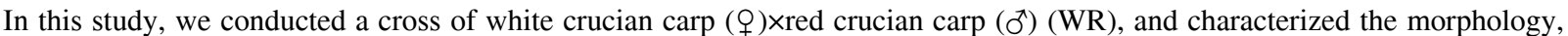
reproduction and genetics of the progeny. Different from parents, WR with the gray color showed the hybrid morphological traits of both parents. WR possessed normal gonads producing mature eggs or sperm, and exhibited high fertilization rate $(90.2 \%)$ and high hatchery rate $(80.5 \%)$, which contributed to produce and enlarge the population. WR with the same DNA content as parents was a diploid fish with 100 chromosomes $(2 n=100)$. Amplified ITS of $45 \mathrm{~S}$ rDNA, in WR the sequences consisting of $884 \mathrm{bp}$ bases of the entire ITS-1 region, $5.8 \mathrm{~S}$ region, and entire ITS- 2 region. The sequences showed high similarity between WR and its parents and leaned towards male inheritance. In WR, NTS of 5S rDNA consisted of three length types with total 654 bp bases. From sequence analysis of NTS, WR shared $94.2 \%$ and $95.1 \%$ similarities with their female and male parent, respectively. Sequence analysis of ITS and NTS revealed that there existed recombination and variation in the hybrid progeny, which was the genetic base for adaptation and speciation. In conclusion, we obtained WR from hybridization and it exhibited hybrid traits in morphology and variation in genetic composition showing essential difference with its parents. The obtainment of WR has important significance in fish genetic breeding.
\end{abstract}

white crucian carp, red crucian carp, hybridization, genomic variation

Citation: Wang J, Xiao J, Zeng M, Xu K, Tao M, Zhang C, Duan W, Liu WB, Luo KK, Liu Y, Liu SJ. Genomic variation in the hybrids of white crucian carp and red crucian carp: evidence from ribosomal DNA. Sci China Life Sci, 2015, 58: 590-601, doi: 10.1007/s11427-015-4835-2

Hybridization including distant crossing (interspecific or higher-ranking taxa hybridization) and intraspecific crossing involves the mating of genetically differentiated individuals or groups to generate new population with heterosis [1-3]. If the progeny derived from hybridization are bisexual and fertile, they can form new species or lineages with genetic variation through self-mating, with major implications for evolutionary biology and breeding. The application of hybrids in aquaculture is an important method and research content in fish genetic breeding. In our previous

$\dagger$ Contributed equally to this work

*Corresponding author (email: 1sj@hunnu.edu.cn) studies, many kinds of fishes were obtained from distant crossing, such as allotetraploid fish lineages derived from Carassius auratus red var. ( $+9 \times$ Cyprinus carpio $\left(\sigma^{\top}\right)$ $\left(\mathrm{F}_{3}-\mathrm{F}_{24}\right)$ [4], autotetraploid fish lineages derived from Carassius auratus red var. (q) $\times$ Megalobrama amblycephala (ठ) [5], diploid fish lineages derived from Megalobrama amblycephala $(0) \times$ Culter alburnus $\left(\mathrm{o}^{\top}\right)\left(\mathrm{F}_{1}-\mathrm{F}_{3}\right)[6]$, different ploidy-level hybrids of Megalobrama amblycephala Yih $\times$ male Xenocypris davidi Bleeke [7] and different ploidy-level hybrids of red crucian carp $\times$ copmouth culter [8]. These hybrid progenies can be used to produce improved triploids or diploids $[9,10]$. In the theory, intraspecific crossing can also produce hybrid lineages with hetero- 
sis, which can be used in production after selection breeding. In aquaculture, there are few examples of establishment and implication of variation lineage by intraspecific crossing. In this study, we conducted a hybridization of white crucian carp (Carassius auratus cuvieri, WCC) and red crucian carp (Carassius auratus red variety, RCC). These are diploid fishes both belonging to Cypriniformes, Cyprinidae (genus Carassius) and with the same chromosome number $(2 n=100)$. WCC is characterized by white body color, high body, small head, short tail, strong reproductive ability, and quick growth. RCC is characterized by red body color, strong stress resistance, and perfect meat quality. However, WCC has the disadvantage of poor meat quality and RCC shows the disadvantage of slow growth. Besides, the red body color of RCC is restricted in terms of consumption. Thus from this crossing, we expect to obtain a new variety that has the combined advantage traits of WCC and RCC. In this study we obtained the hybrids of WR $\left(\mathrm{F}_{1}\right)$ from white crucian carp by red crucian carp and $\mathrm{F}_{2}$ from $\mathrm{F}_{1}$ inbred.

In this work, we characterized the morphological characteristics (countable and measurable traits) and gonad development of the hybrids. In addition, we investigated the cytogenetics of DNA content and chromosome number, and the molecular genetics of two multigene families 5S rDNA and ITS (from 45S rDNA) of WR. The ribosomal genes are divided into two clusters: the major (45S rDNA) and the minor (5S rDNA) clusters. The $45 \mathrm{~S}$ rDNA has two internal transcribed spacers (ITS1 and ITS2), which are rapidly evolving DNA regions with high frequency of nucleotide mutations which make them useful as species-specific molecular markers. Meanwhile, the 5S rDNA comprises a conserved coding region of $120 \mathrm{bp}$ and a non-transcribed spacer (NTS) which is variable in length and sequence among species. The two ribosomal clusters represent well-known examples of concerted evolution.

The results showed that the morphological traits of WR were intermediate between the paternal and maternal traits. The body color of WR was gray without red or white. WR possessed normal gonadal development. In cytogenetics level, the results showed $2 n$ WR with 100 chromosomes. Sequence analysis of NTS and ITS revealed that gene recombination and base mutation happened in the hybrid progeny compared to its parents, which was the base for establishment of variation lineage. The heterosis of WR was just the needs of fish breeding. The formation and characterization of WR is important in biological evolution and fish genetic breeding.

\section{Materials and methods}

The samples including WCC and RCC were obtained from the Engineering Research Center of Polyploid Fish Breeding and Reproduction of Ministry of Education at Hunan
Normal University.

\subsection{Animals and crosses}

During the reproductive seasons (from April to June), mature parental fishes were selected. The WR was formed by crossing haploid egg of WCC with haploid sperm of RCC. The progeny of reciprocal cross $\left(\mathrm{RCC}(q) \times \mathrm{WCC}\left({ }^{\top}\right), \mathrm{RW}\right)$ was formed by crossing haploid egg of RCC with haploid sperm of WCC. Mature eggs were fertilized and the embryos developed in the culture dishes at the water temperature of $20-22^{\circ} \mathrm{C}$. About 4,000 embryos were taken at random for the examination of the fertilization rate (number of embryos at the stage of gastrula/number of fertilized eggs $\times 100 \%$ ) and hatching rate (number of hatched fry/number of fertilized eggs $\times 100 \%)$. The hatched fry were transferred to the pond for further culture.

\subsection{Measurement of morphological traits}

We randomly selected 20 one-year-old fishes from each group (WCC, RCC, WR and RW) for morphological examination. We measured total length, body length and height, head length and height, and caudal peduncle length and height (accurate to $0.1 \mathrm{~cm}$ ) in each fish. These values were converted to ratios. In addition, we recorded counts of the following variables: number of lateral scales, number of upper and lower lateral scales, number of dorsal fins, number of abdominal fins, and number of anal fins. These data were analyzed by SPSS.

\subsection{Examination of the ploidy level}

We measured the DNA content of $20 \mathrm{WR}$ and $20 \mathrm{RW}$ individuals. The diploid WCC and RCC were used as control groups. We collected $0.5-1 \mathrm{~mL}$ blood from the caudal vein of each individual using a syringe containing $200-400 \mathrm{U}$ sodium heparin. The blood samples were then filtered, and stained with Cystain DNA 1 Step Staining Solution (Partec, Görlitz, Germany). The DNA content of each sample was measured by flow cytometry (Partec). The method of chromosome preparations referred to Luo [11].

\subsection{Observation of the gonadal structure}

To observe the gonadal structure, we selected eight 10-month-old WR and RW. The gonads were fixed in Bouin's solution for $24 \mathrm{~h}$, then anhydrated using an ethanol gradient and transparentized in xylene. The sections were embedded in paraffin, cut at 6-8 $\mu \mathrm{m}$, and stained with hematoxylin and eosin. The microstructure was observed and photographed using a Pixera Pro 600ES. We identified the gonad stages based on standards for cyprinid fish [12]. 


\subsection{Genomic DNA extraction, PCR and sequencing}

Total genomic DNA were extracted from the peripheral blood cells of RCC, WCC, RW and WR using a phenol/chloroform extraction method as described in Sambrook et al. [13]. Two sets of primers were used as described by Masaru Murakami [14]. 5S rDNA F: 5'-GCTATGCCCGATCTCGTCTGA-3' and R: 5'-CAGGTTGGTATGGCCGTAAGC-3'; ITS F: 5'-AGTCGTAACAAGGTTTCCGTAG$3^{\prime}$ and R: 5'-GCTTA (G/C/A)TAATATGCTTAAATTC-3') were designed and synthesized to amplify the 5S rDNA and ITS from genomic DNA. The amplification reaction mixture $(25 \mu \mathrm{L})$ consisted of $20 \mathrm{ng}$ genomic DNA, $1.5 \mathrm{mmol} \mathrm{L}^{-1}$ $\mathrm{MgCl}_{2}, 0.2 \mathrm{mmol} \mathrm{L}^{-1}$ of each dNTP, $0.4 \mu \mathrm{mol} \mathrm{L}{ }^{-1}$ of each primer, $1 \times$ PCR buffer, and $1.25 \mathrm{U}$ Taq polymerase (TaKaRa, Dalian, China). The thermal program consisted of an initial denaturation step of $94^{\circ} \mathrm{C}$ for $5 \mathrm{~min}$, followed by 30 cycles $\left(94^{\circ} \mathrm{C}\right.$ for $30 \mathrm{~s}, 59^{\circ} \mathrm{C}$ for $30 \mathrm{~s}$, and $72^{\circ} \mathrm{C}$ for $5 \mathrm{~min}$ ) and a final extension step of $72^{\circ} \mathrm{C}$ for $10 \mathrm{~min}$. The PCR products were separated on a $1.2 \%$ agarose gel, purified using a Gel Extraction Kit ((Sangon Biotech Co., Ltd., Shanghai, China), ligated into a pMD18-T vector, and transferred into E. coli DH5 $\alpha$. The positive clones were then sequenced (10 clones for each PCR fragment). To determine sequence homology and variation, the sequences were aligned using BioEdit [15] and Clustal W [16].

\section{Results}

\subsection{Fertilization rates and hatching rates}

In the reciprocal crossing and self-crossing of RCC and WCC, we observed high fertilization rates (WCC $(q) \times \mathrm{RCC}$ (ठ): $90.2 \%, \operatorname{RCC}(q) \times \mathrm{WCC}\left(\sigma^{\top}\right): 90.0 \%, \mathrm{RCC}(q) \times \mathrm{RCC}$

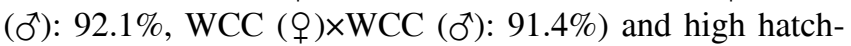
ing rates $\left(\mathrm{WCC}(q) \times \mathrm{RCC}\left(\sigma^{\top}\right): 80.5 \%, \mathrm{RCC}(q) \times \mathrm{WCC}\left(\sigma^{\top}\right)\right.$ : $82.3 \%, \operatorname{RCC}(q) \times \operatorname{RCC}\left(\sigma^{\top}\right): 84.5 \%$, WCC $(q) \times \mathrm{WCC}\left(\sigma^{\top}\right):$ $82.5 \%)$. There was no significant difference in fertilization or hatching rates between the hybrid groups and the parental self-crossing groups $(P>0.05)$.

\subsection{Comparison of morphological traits among RCC, WCC, RW, and WR}

The hybrid progenies were all gray in body color with no red color (Figure 1). We produced $\mathrm{F}_{2}$ which were all gray in body color without color differentiation. In this study we charged measurable characteristics into countable characteristics, reducing the influence caused by different size of fish. Table 1 presents the countable traits observed in WR, RW, WCC and RCC. Table 2 presents the measurable characteristics of WR, RW, WCC and RCC. RW and WR exhibited traits that were intermediate between the paternal and maternal traits.
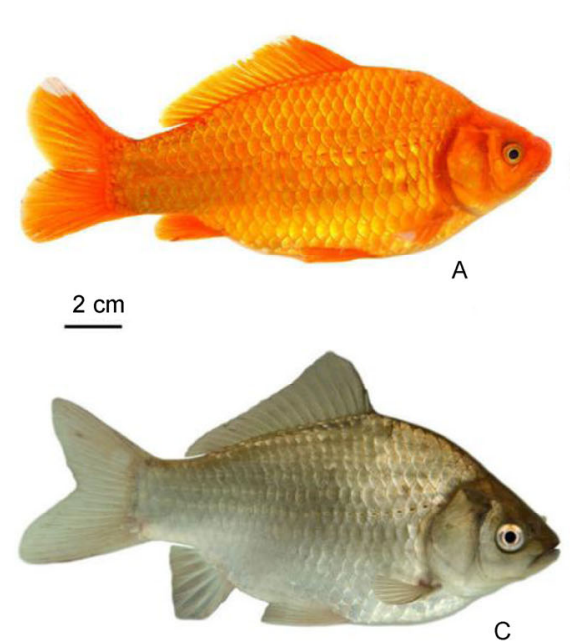

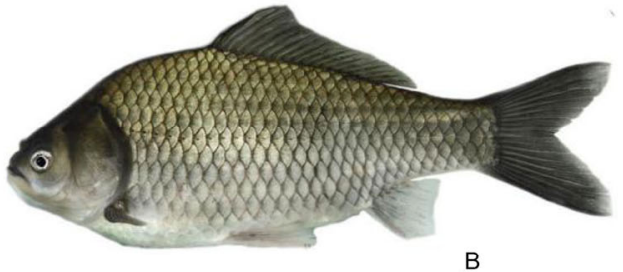

B

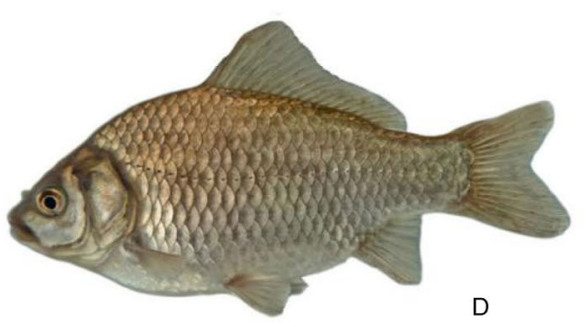

Figure 1 Images of RCC (A), WCC (B), RW (C), and WR (D).

Table 1 The ratios of measurable traits of RCC, WCC, WR and RW

\begin{tabular}{ccccccc}
\hline Fish type & $\begin{array}{c}\text { Total length/ } \\
\text { body length }\end{array}$ & $\begin{array}{c}\text { Body length/ } \\
\text { body height }\end{array}$ & $\begin{array}{c}\text { Body length/ } \\
\text { head length }\end{array}$ & $\begin{array}{c}\text { Head length/ } \\
\text { head height }\end{array}$ & $\begin{array}{c}\text { Caudal peduncle length/ } \\
\text { caudal peduncle height }\end{array}$ & $\begin{array}{c}\text { Head height/ } \\
\text { caudal peduncle height }\end{array}$ \\
\hline WCC & $1.24 \pm 0.02$ & $2.22 \pm 0.15$ & $3.70 \pm 0.21$ & $1.17 \pm 0.06$ & $0.81 \pm 0.01$ & $1.78 \pm 0.09$ \\
RCC & $1.23 \pm 0.02$ & $2.20 \pm 0.16$ & $3.72 \pm 0.27$ & $1.18 \pm 0.04$ & $0.79 \pm 0.02$ & $1.80 \pm 0.07$ \\
WR & $1.25 \pm 0.03$ & $2.20 \pm 0.15$ & $3.70 \pm 0.25$ & $1.19 \pm 0.06$ & $0.82 \pm 0.02$ & $1.81 \pm 0.12$ \\
RW & $1.24 \pm 0.03$ & $2.23 \pm 0.17$ & $3.69 \pm 0.31$ & $1.19 \pm 0.07$ & $0.82 \pm 0.02$ & $1.82 \pm 0.10$ \\
\hline
\end{tabular}




\subsection{Ploidy levels of the hybrids}

DNA content of WCC and RCC were used as control. The flow cytometry results indicated that the DNA content of the hybrids was the same as that of parental $(P>0.05)$ (Figure 2), implying that these hybrids were diploid fish. Figure 3 shows the chromosome metaphase spreads. RW and WR possessed 100 chromosomes, indicating that they were diploid $(2 n=100)$.

\subsection{Analysis of gonadal development of the hybrids}

Histological analysis revealed that gonadal development in the hybrid was synchronized. Figure 4 presents the gonadal microstructure of 10-month-old WR and RW. The ovaries developed normally and were mainly composed of oocytes at phase IV-stage (Figure 4A and B). The testes of the hybrids were full of mature sperm (Figure 4C and D). Both RW and WR were sexually mature at one-year-old. White sperm or mature ova could be stripped out from 12-monthold hybrids.

\subsection{Analysis of 5S rDNA and ITS}

PCR amplification with 5S primers for RCC, WCC, WR and RW produced the same pattern with three bands (Figure 5). Sequencing revealed the DNA fragments to be 203, 340, and $471 \mathrm{bp}$ in length. Using BLASTn, all fragments were confirmed to be 5S rDNA repeat units, each comprising a $3^{\prime}$ end of the coding region (positions 1-21), a whole NTS region, and a large 5' coding region of the adjacent unit (positions 22-120). Three fragments of 5S rDNA (designated class I: 203 bp; class II: 340 bp; and class III: 471 bp) were categorized into different NTS types (designated NTS-I, II, and III for the 83, 220, and 351 bp monomers, respectively). The sequence data revealed that in the $5 \mathrm{~S}$ coding regions, the parental samples RCC and WCC show species-specific nucleotide sites (position-22: RCC-G, WCC-T and position-

Table 2 Morphological assessment of RCC, WCC, WR and RW

\begin{tabular}{|c|c|c|c|c|c|c|}
\hline Fish type & Lateral scale & Upper lateral scale & Lower lateral scale & Back fin & Abdominal fin & Anal fin \\
\hline WCC & $32-34$ & $6-8$ & $5-7$ & III+18-20 & $8-10$ & $\mathrm{III}+6-7$ \\
\hline $\mathrm{RCC}$ & $28-30$ & $5-6$ & $6-7$ & $\mathrm{III}+18-20$ & $8-9$ & $\mathrm{III}+6-7$ \\
\hline WR & $30-32$ & $5-7$ & $6-7$ & $\mathrm{III}+18-20$ & $8-10$ & $\mathrm{III}+6-7$ \\
\hline RW & $30-32$ & $6-8$ & $5-7$ & III $+18-20$ & $8-10$ & III $+6-7$ \\
\hline
\end{tabular}
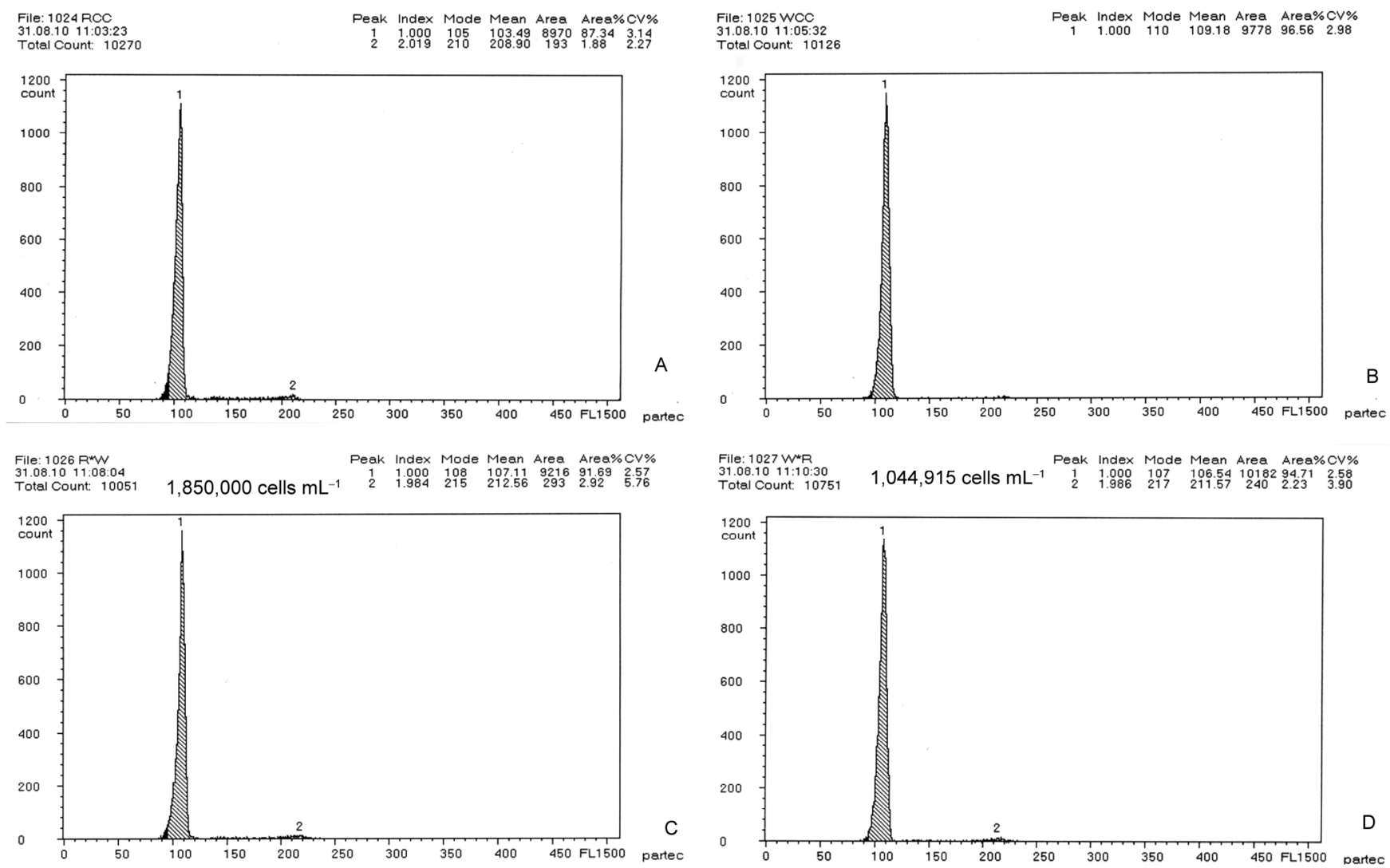

Figure 2 The DNA content in RCC (A), WCC (B), RW (C), and WR (D). 

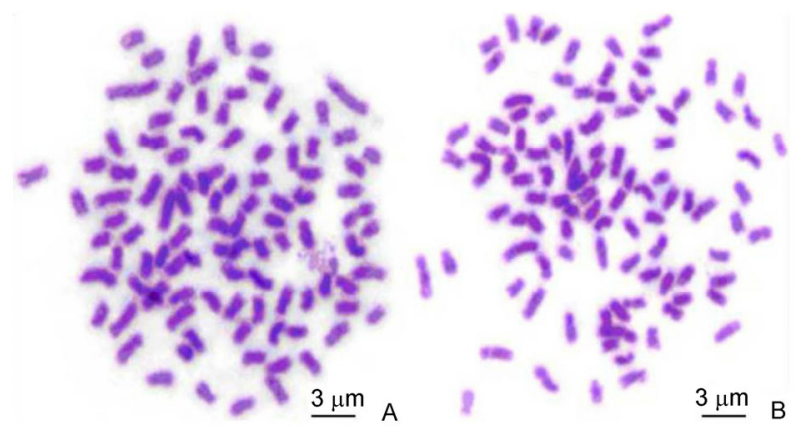

Figure 3 The metaphase chromosome spreads of RW and WR. A, The metaphase chromosome spread of RW $(2 n=100)$. B, The metaphase chromosome spread of WR $(2 n=100)$.

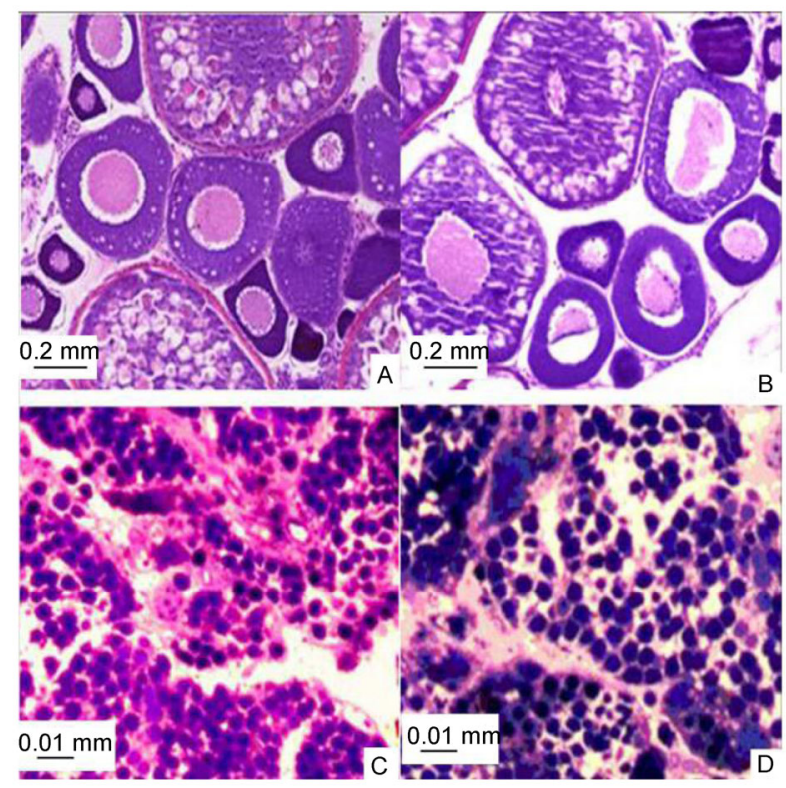

Figure 4 Gonad microstructure of RW and WR. The mature ovary of RW (A) and WR (B). The mature testis of RW (C) and WR (D).

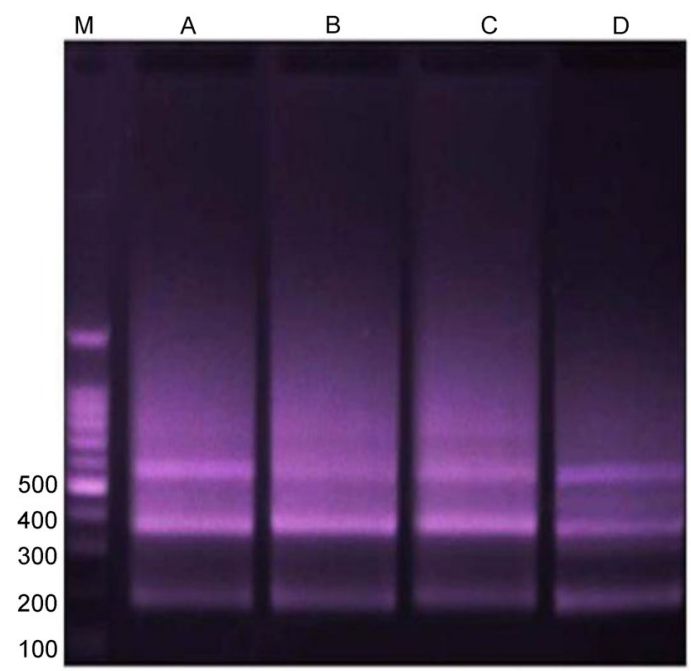

Figure 5 DNA bands amplified from RCC, WCC, RW, and WR. M: DNA 100 bp ladder; 5S amplification resulted in three DNA bands ( 200, 350 and $500 \mathrm{bp}$ ) from RCC (A), WCC (B), RW (C), and WR (D).
66: RCC-G, WCC-A). The progenies RW and WR inherited at position-22 the WCC nucleotide with $\mathrm{T}$ and at position-66 their respective male-parent nucleotide (RCC: G, WCC: A, RW: A, WR: G) (Figure 6). In the full length of NTS, the sequence similarities of WR with WCC and RCC were $94.2 \%$ and $95.1 \%$, respectively. Comparative analysis of the NTS between the hybrid offspring and their parents showed obvious recombination and base mutation. In NTS-83, the nucleotide of WR was different from its parents at seven sites (position-5, -9, -12, -17, -24, -25 and -33) (Figure 7), revealing large variations. Table 3 shows the bases in the hybrid offspring that were different from the parents. In the NTS-220, sequence from the hybrid progenies exhibited influence by RCC (Figure 8), whereas in the sequence NTS-351, sequence from the hybrid progenies exhibited influence by WCC (Figure 9) and WR displayed larger variation than RW (Table 3).

PCR amplification, sequencing and sequences analysis of the $45 \mathrm{~S}$ rDNAs of WCC, RCC, WR and RW revealed that these were of the same length (884 bp) with ITS1 (341 bp)+5.8S (159 bp)+ITS2 (384 bp) showing above 99\% sequence similarity (Table 4). In the 5.8S rDNA, we observed one nucleotide difference at position -158 (RCC: T, WCC: C, RW: C, WR: T) (Figure 10). In ITS1 region, we observed two nucleotide differences at positions-90 (RCC: A, WCC: C, RW: C, WR: C) and -309 (RCC: A, WCC: G, RW: G, WR: A) (Figure 11). In ITS-2 region, we observed four nucleotide differences at positions-141 (RCC: T, WCC: -, RW: -, WR: -), -325 (RCC: -, WCC: -, RW: -, WR: T), -349 (RCC: -, WCC: C, RW: C, WR: -) and -351 (RCC: C, WCC: A, RW: A, WR: C) (Figure 12). In Table 4, the hereditary characteristics of ITS were inclined to paternal inheritance.

\section{Discussion}

Hybridization, as a means of species modification, plays a very important part in the speciation and improvement of animals [17-19]. It integrates the genetic material of both parent species into one single group, resulting in changes in the gene regulation and expression in the hybrids [18,20,21].

Table 3 Similarity of NTS sequences between hybrids and their parents

\begin{tabular}{ccccc}
\hline NTS type & WR/WCC & WR/RCC & RW/RCC & RW/WCC \\
\hline NTS-83 bp & $91.6 \%$ & $89.2 \%$ & $98.8 \%$ & $98.8 \%$ \\
NTS-220 bp & $94.5 \%$ & $99.1 \%$ & $99.1 \%$ & $94.5 \%$ \\
NTS-351 bp & $98.0 \%$ & $96.9 \%$ & $96.3 \%$ & $99.1 \%$ \\
\hline
\end{tabular}

Table 4 Similarity of ITS sequences between hybrids and their parents

\begin{tabular}{ccccc}
\hline ITS type & WR/WCC & WR/RCC & RW/RCC & RW/WCC \\
\hline ITS-1 & $99.7 \%$ & $99.7 \%$ & $99.4 \%$ & $100 \%$ \\
ITS-2 & $99.2 \%$ & $99.5 \%$ & $99.2 \%$ & $100 \%$ \\
\hline
\end{tabular}




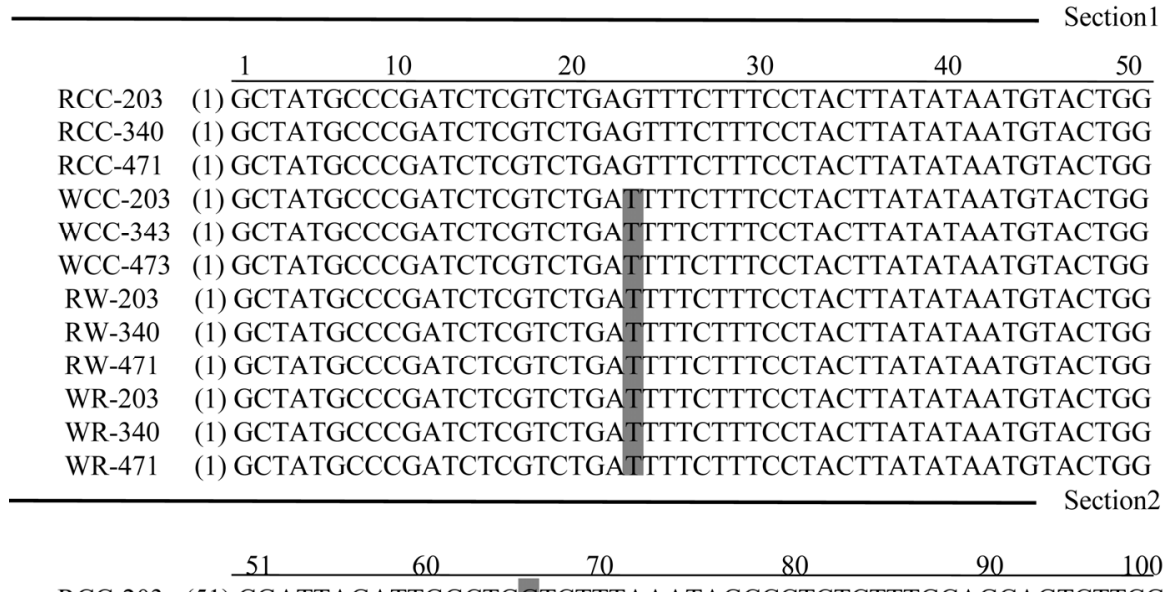

RCC-203 (51) CGATTAGATTGGCTGGTCTTTAAATAGCCCTCTCTTTGCAGCAGTCTTCG RCC-340 (51) CGATTAGATTGGCTGGTCTTTAAATAGCCCTCTCTTTGCAGCAGTCTTCG RCC-471 (51) CGATTAGATTGGCTGGTCTTTAAATAGCCCTCTCTTTGCAGCAGTCTTCG WCC-203 (51) CGATTAGATTGGCTGATCTTTAAATAGCCCTCTCTTTGCAGCAGTCTTCG WCC-343 (51) CGATTAGATTGGCTGATCTTTAAATAGCCCTCTCTTTGCAGCAGTCTTCG WCC-473 (51) CGATTAGATTGGCTGATCTTTAAATAGCCCTCTCTTTGCAGCAGTCTTCG RW-203 (51) CGATTAGATTGGCTGATCTTTAAATAGCCCTCTCTTTGCAGCAGTCTTCG RW-340 (51) CGATTAGATTGGCTGATCTTTAAATAGCCCTCTCTTTGCAGCAGTCTTCG RW-471 (51) CGATTAGATTGGCTGATCTTTAAATAGCCCTCTCTTTGCAGCAGTCTTCG WR-203 (51) CGATTAGATTGGCTGGTCTTTAAATAGCCCTCTCTTTGCAGCAGTCTTCG WR-340 (51) CGATTAGATTGGCTGGTCTTTAAATAGCCCTCTCTTTGCAGCAGTCTTCG WR-471 (51) CGATTAGATTGGCTGGTCTTTAAATAGCCCTCTCTTTGCAGCAGTCTTCG Section3

$\begin{array}{lc}\frac{101}{n} \text { RCC-203 } & 11010 \\ \text { RCC-340 } & \text { CTTACGGCCATACCAACCTG } \\ \text { RCC-471 } & \text { CTTACGGCCATACCAACCTG } \\ \text { WCC-203 (101) CTTACGGCCATACCAACCTG } & \text { CTACGCCATACCAACCTG } \\ \text { WCC-343 (101) CTTACGGCCATACCAACCTG } \\ \text { WCC-473 (101) CTTACGGCCATACCAACCTG } \\ \text { RW-203 (101) CTTACGGCCATACCAACCTG } \\ \text { RW-340 (101) CTTACGGCCATACCAACCTG } \\ \text { RW-471 (101) CTTACGGCCATACCAACCTG } \\ \text { WR-203 (101) CTTACGGCCATACCAACCTG } \\ \text { WR-340 (101) CTTACGGCCATACCAACCTG } \\ \text { WR-471 (101) CTTACGGCCATACCAACCTG }\end{array}$

Figure 6 Comparison of the 5S coding regions from RCC, WCC, RW, and WR.

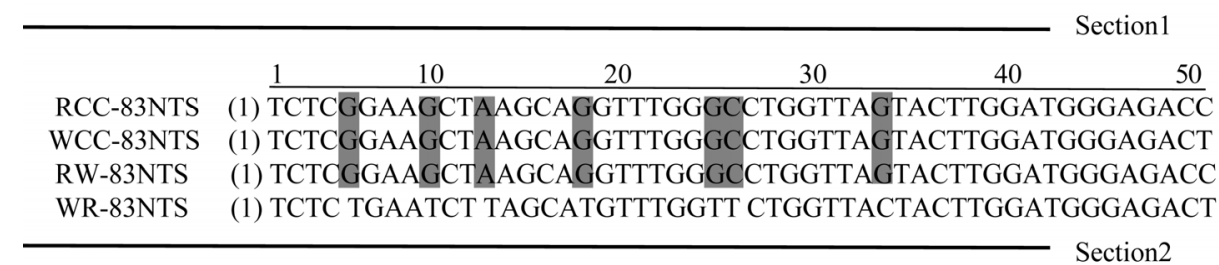

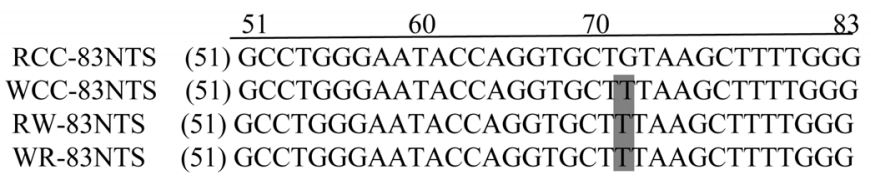

Figure 7 Comparison of the NTS-83 sequences from RCC, WCC, RW, and WR.

Thus, the hybrids gain heterosis in many aspects, such as growth rate, desirable traits, harvestability, and environmental tolerances $[1,22]$. The two kinds of hybridization are distant crossing (above-specific or interspecific crossing) and intraspecific crossing. Through long-term and systematic research in distant hybridization, our lab cultivated a lot 


\begin{tabular}{lcccc} 
& \multicolumn{1}{c}{10} & 20 & 30 & 40 \\
RCC-220NTS & $(1)$ TCTCGGAAGCTAAGCAGGTTTGGGCCTGGTTAGTACTTGGATGGGAGACC \\
WCC-220NTS & $(1)$ TCTCGGAAGCTAAGCAGGTTTGGGCCTGGTTAGTACTTGGATGGGAGACC \\
RW-220NTS & (1) TCTCGGAAGCTAAGCAGGTTTGGGCCTGGTTAGTACTTGGATGGGAGACC \\
WR-220NTS & (1) TCTCGGAAGCTAAGCAGGTTTGGGCCTGGTTAGTACTTGGATGGGAGACC \\
\hline
\end{tabular}

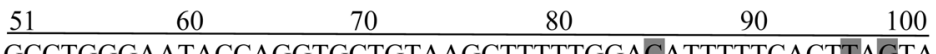

RCC-220NTS (51) GCCTGGGAATACCAGGTGCTGTAAGCTTTTTGGACATTTTTCACTTAGTA

WCC-220NTS (51) GCCTGGGAATACCAGGTGCTGTAAGCTTTTTGGAAATTTTTCACTAATTA

RW-220NTS (51) GCCTGGGAATACCAGGTGCTGTAAGCTTTTTGGACATTTTTCACTTAGTA WR-220NTS (51) GCCTGGGAATACCAGGTGCTGTAAGCTTTTTGGACATTTTTCACTTAGTA

$\longrightarrow$ Section3

$\begin{array}{lcccc} & 101 & 110 & 120 & 130 \\ \text { RCC-220NTS } & (101) \text { TATAATAATTTTGCCAAAAAATAGAGTCAATGCCCGATCTCTGAATCTTA } \\ \text { WCC-220NTS } & (101) \text { TATAATAATTGTGCCAAAAAAAAGAGTCAATGCCCGATCTCTGAATCTTA } \\ \text { RW-220NTS } & (101) \text { TATAATAATTTTGCCAAAAAATAGAGTCAATGCCCGATCTCTGAATCTTA } \\ \text { WR-220NTS } & (101) \text { TATAATAATTTTGCCAAAAAATAGAGTCAATGCCCGATCTCTGAATCTTA }\end{array}$

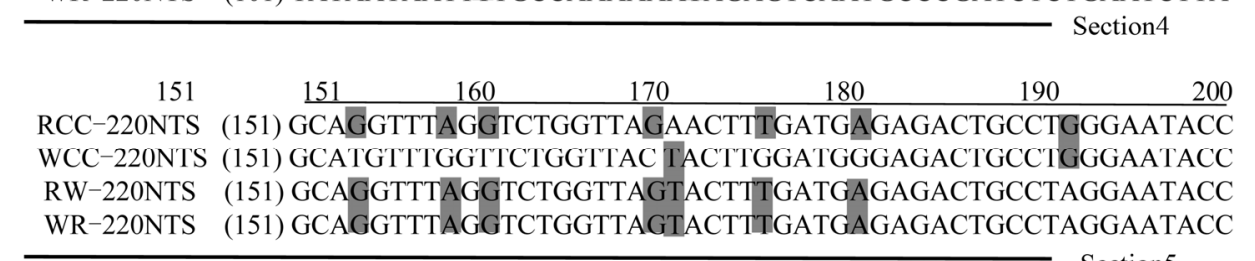

\begin{tabular}{|c|c|c|}
\hline & 201 & 220 \\
\hline RCC-220NTS & (201) AGGTGCTTTAAC & GGG \\
\hline WCC-220NTS & (201) AGGTGCTTTAAC & GGG \\
\hline RW-220NTS & (201) AGGTGCTTTAAC & TGGG \\
\hline WR-220NTS & (201) AGGTGCTTTAAC & ГGGG \\
\hline
\end{tabular}

Figure 8 Comparison of the NTS-220 sequences from RCC, WCC, RW, and WR.

of excellent new strains successfully, such as allotetraploid fish lineages derived from Carassius auratus red var. $($ $) \times$ Cyprinus carpio $\left({ }^{\top}\right)\left(\mathrm{F}_{3}-\mathrm{F}_{24}\right)$, autotetraploid fish lineages derived from Carassius auratus red var. $(q) \times$ Megalobrama amblycephala (ठ), diploid fish lineages derived from Megalobrama amblycephala $(q) \times$ Culter alburnus $(\widetilde{\sigma})\left(\mathrm{F}_{1}-\mathrm{F}_{3}\right)$. At the DNA level, distant hybridization leads to the emergence of recombinant DNA variation in the offspring. With regard to phenotype, distant hybridization integrates the advantages of both parents, so that heterosis of shape, growth rate, survival, disease resistance, and other traits is exhibited by future generations [23]. An in-depth study of fish chromosome evolution, unreduced gamete formation mechanism, polyploidy, gene recombination and variation can be continued on the basis of the distant hybridization above. Distant hybridization offspring have broader applications in production, such as the production of new polyploid fish, sterile triploid fish and the new diploid fish. The formation of distant hybrid lineages is very useful for studies of biological evolution and genetic breeding.

Intraspecific hybridization defined as a cross between two subspecies or different strains in same species. Because of the close relationship, hybrid lethality and intersterility will not appear in intraspecific hybridization. Although intraspecific hybridization brings finite genetic variation than distant hybridization in hybrids, as long as matches appropriately, intraspecific hybridization can also produce the offspring possessing the excellent traits generated from parents. Furthermore, it is easy for intraspecific hybridization to build a steady lineage. The genetically stable hybrids can not only transmit the heterosis generation by generation, but also produce various new strains or improve fish by match with the same or different ploidy fish. Thus, intraspecific crossing is indispensable to fish genetic improvement.

The choice of parents for hybridization experiments requires consideration of chromosome number, phylogenetic relationships, reproductive characteristics, shape, diet, growth rate, disease resistance, body color and other factors. When designing hybrid crossing, parents possessing different desirable traits are chosen to produce hybrids with a combination of excellent hybrid characteristics. In this paper, we carried out the crossing of white crucian carp and red crucian carp which possess the same number of chromosomes, different color, and different excellent traits. Both of white crucian carp and red crucian carp belong to the order Cypriniformes, genus Carassius. Previously, white 


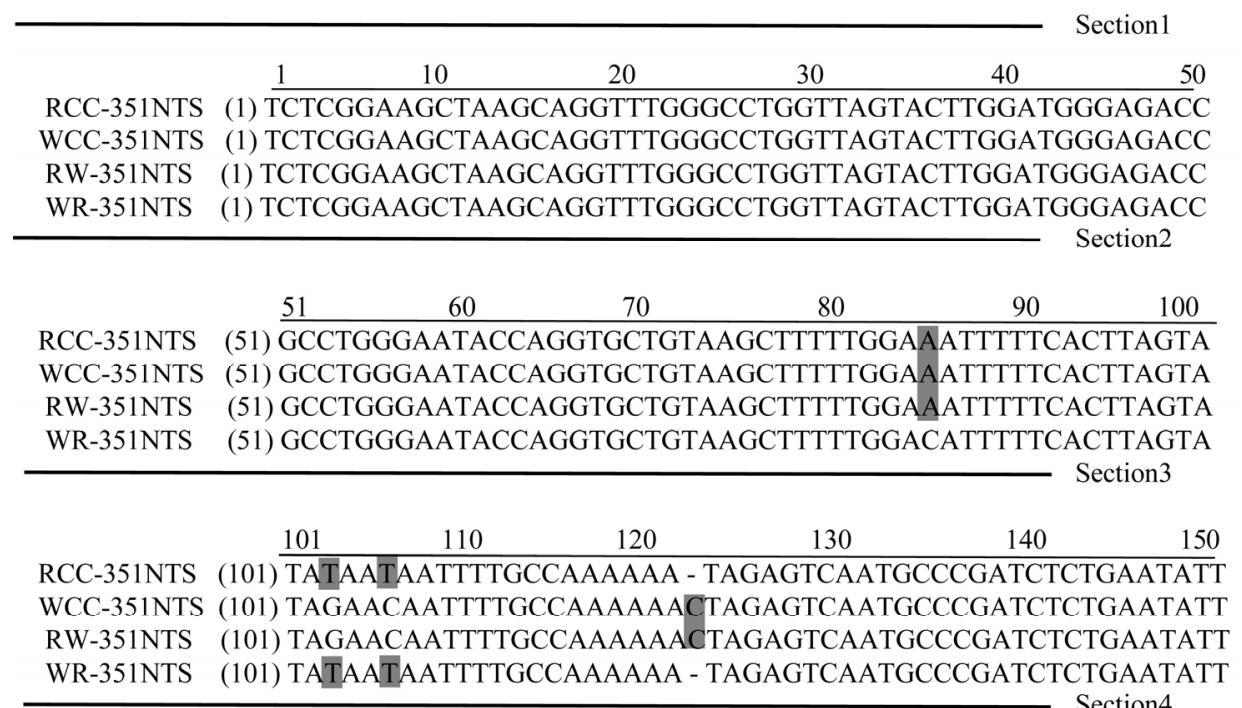

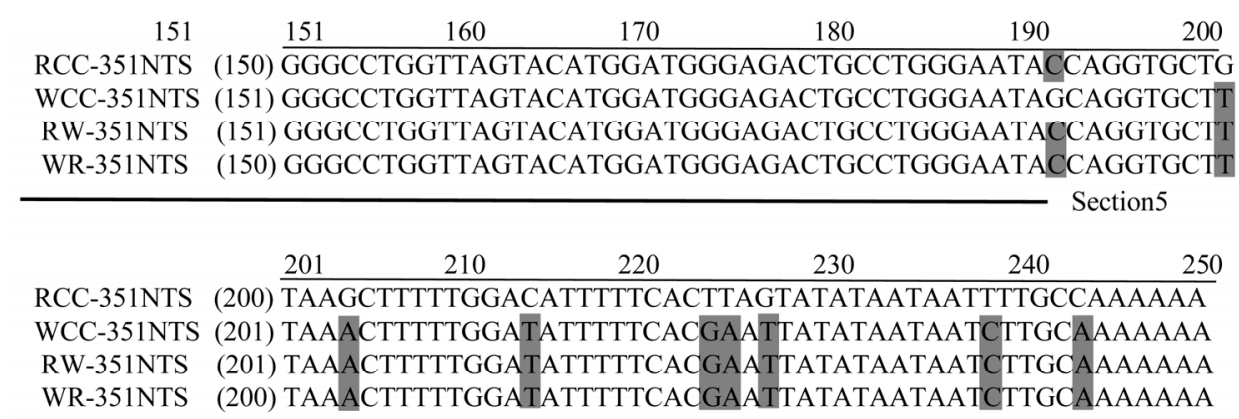
WR-35INTS (200) TAA

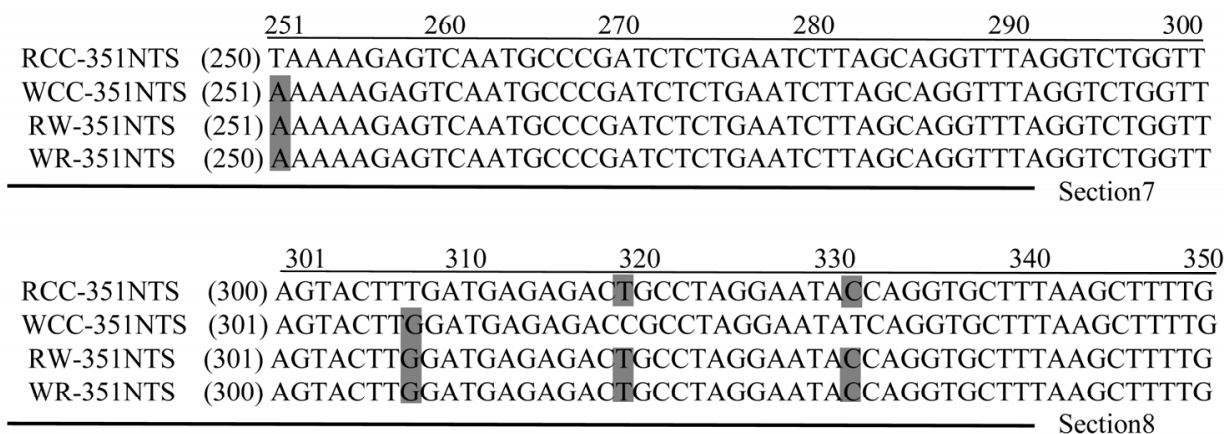

$\begin{array}{lr}\text { RCC-351NTS } & (350) \underline{\frac{351}{G G}} \\ \text { WCC-351NTS } & (351) \mathrm{GG} \\ \text { RW-351NTS } & (351) \mathrm{GG} \\ \text { WR-351NTS } & (350) \mathrm{GG}\end{array}$

Figure 9 Comparison of the NTS-351 sequences from RCC, WCC, RW and WR.

crucian carp had been treated as a species, C. Carassius, the same as red crucian carp. However, based on ample molecular evidence recently, white crucian carp is considered to be an independent species, Carassius cuvieri [24-26]. Therefore, the crossing of white crucian carp and red crucian carp can also be considered as a distant hybridization at the interspecific level. The degree of heredity and heterosis was assessed from shape, fertilization rate, hatching rate, gonadal development, ploidy level, 5S rDNA and ITS of parental fishes and hybrids. In this hybridization, we observed phenotypic alteration in the hybrids. The hybrids' measurable characteristics and countable characteristics are between parents'. The hybrid progenies were all gray in body color without red color. The hybrids exhibited high fertilization and hatching rates, which were close to that of the parental self-mating. The hybrids all reach sexual ma- 


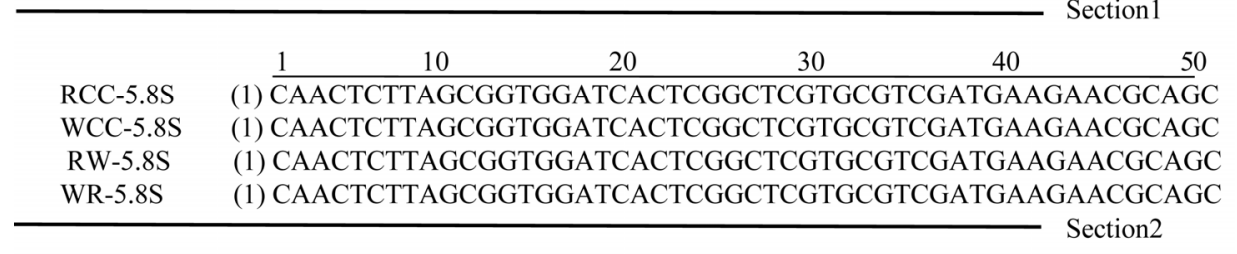

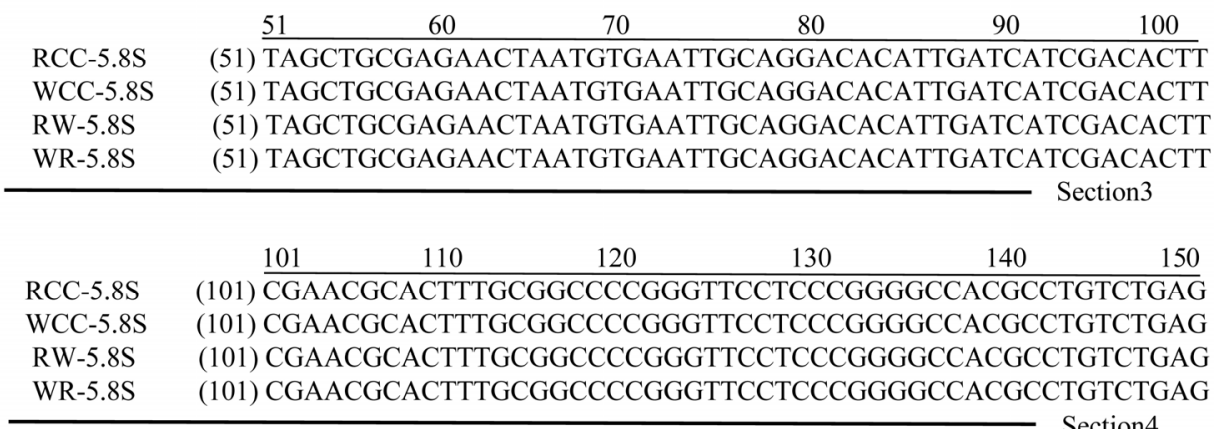

$\begin{array}{lc} & 151 \quad 159 \\ \text { RCC-5.8S } & \text { (151) GGTCGCTTT } \\ \text { WCC-5.8S } & \text { (151) GGTCGCTCT } \\ \text { RW-5.8S } & \text { (151) GGTCGCTCT } \\ \text { WR-5.8S } & \text { (151) GGTCGCTTT }\end{array}$

Figure 10 Comparison of the 5.8s rDNA sequences from RCC, WCC, RW, and WR.

Table 5 Nucleotide changes in NTS and ITS ${ }^{\text {a) }}$

\begin{tabular}{|c|c|c|c|c|c|c|}
\hline Fish type & Sequence type & Sequence length (bp) & Change base\# & Mutation base\# & Female-specific base\# & Male-specific base\# \\
\hline \multirow{3}{*}{ RW } & NTS-83 & 83 & 2 & 0 & 1 & 1 \\
\hline & NTS-220 & 220 & 13 & 1 & 11 & 1 \\
\hline & NTS-351 & 351 & 16 & 0 & 3 & 13 \\
\hline \multirow{5}{*}{ WR } & Total & & 31 & 1 & 15 & 15 \\
\hline & NTS-83 & 83 & 9 & 7 & 2 & 0 \\
\hline & NTS-220 & 220 & 13 & 1 & 1 & 11 \\
\hline & NTS-351 & 351 & 17 & 1 & 10 & 6 \\
\hline & Total & & 39 & 9 & 13 & 17 \\
\hline \multirow{2}{*}{ RW } & ITS-1 & 341 & 2 & 0 & 0 & 2 \\
\hline & ITS-2 & 384 & 3 & 0 & 0 & 3 \\
\hline \multirow{4}{*}{ WR } & Total & & 5 & 0 & 0 & 5 \\
\hline & ITS-1 & 341 & 2 & 0 & 1 & 1 \\
\hline & ITS-2 & 384 & 4 & 1 & 1 & 2 \\
\hline & Total & & 6 & 1 & 2 & 3 \\
\hline
\end{tabular}

a) \# represents number.

turity in one year, and WR had been successfully developed $\mathrm{F}_{2}$ generation, which charged the traditional view of distant hybrids' infertility or sterility. This was an advantage impossible in other distant crossing. Flow cytometry and chromosome preparation confirmed that the hybrids are diploid fish without polyploid progeny. This is different from some interspecific crossing which results in polyploidization in the hybrid progeny $[3,4,27]$.

In molecule genetics, ribosomal DNA was studied. The moderate conserved regions of NTS and ITS show quick evolution speed, and these are used to analyze evolution and genetic relationship. In this study, the sequence of NTS in the hybrids of WR and RW exhibited obvious changes that can be categorized into three: (i) hybrids heredity female parent (15 sites in RW and 13 sites in WR), (ii) heredity male parent (15 sites in RW and 17 sites in WR), and (iii) base mutated (seven sites in WR-NTS-83, one site in WR/RW-NTS-220, one site in WR-NTS-351) (Table 5). This indicated that the hybridization resulted in gene recombination and base mutation in the hybrid progeny. ITS 


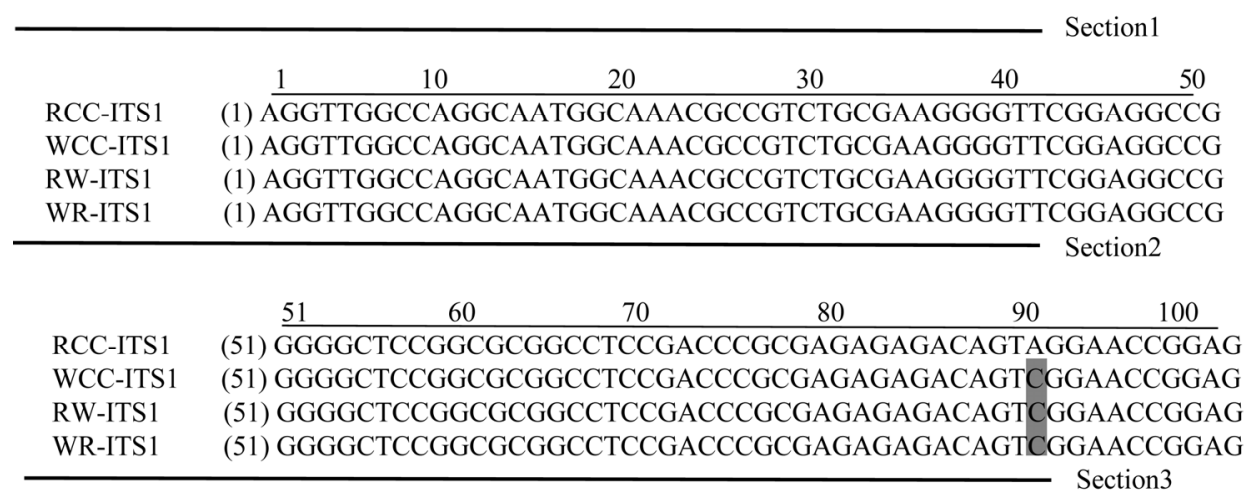

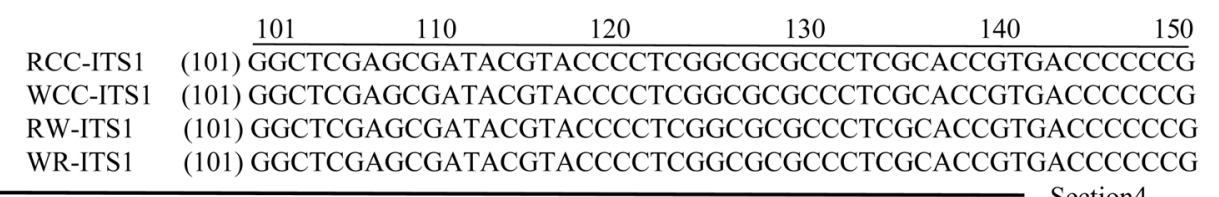

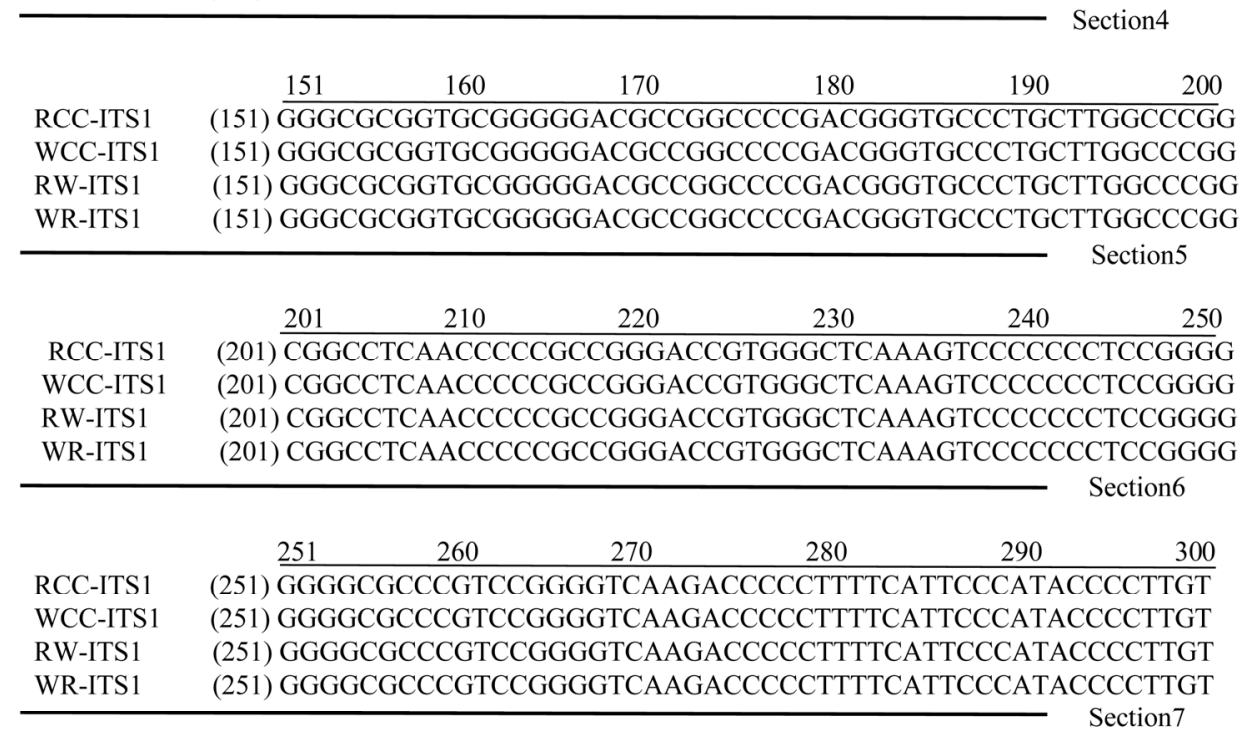

$\begin{array}{ll} & 301 \\ \text { RCC-ITS1 } & \text { (301) CTGCGGCTAAAGGCCTCGATACCTCTAACAAAAAAAGAGTA } \\ \text { WCC-ITS1 } & \text { (301) CTGCGGCTGAAGGCCTCGATACCTCTAACAAAAAAAGAGTA } \\ \text { RW-ITS1 } & \text { (301) CTGCGGCTGAAGGCCTCGATACCTCTAACAAAAAAAGAGTA } \\ \text { WR-ITS1 } & \text { (301) CTGCGGCTAAAGGCCTCGATACCTCTAACAAAAAAAGAGTA }\end{array}$

Figure 11 Comparison of the ITS1 sequences from RCC, WCC, RW, and WR.

sequence analysis also showed that genetic recombination and base mutation happened in WR. Table 5 lists the base variation in NTS and ITS of WR and RW. Recombination of nuclear DNA in hybrids plays an important role in hybrid fish lineages formation and molecular evolution, which is one of the important genetic variation characteristics. It makes the hybrids adapt to the environment, survive and multiply sustainably in the hybrid genetic background. In both NTS and ITS, the base variation in WR showed more frequently than that in RW (Table 5). RW exhibited $100 \%$ similarity in ITS sequence with the male parent of WCC. In WR, there were three types of specific base observed (female parent specific base, male parent specific base and mutation base), leaning towards paternal inheritance.
In morphology, there was no significant difference between WR and RW. At the genomic level, however, significant differences became apparent in WR showing larger variation than RW. The reason and more various genetic traits of WR are being researched. Nuclear DNA mutation and recombination were detected in WR, which was the basis for phenotypic changes. WR inherited both female advantages like rapid growth rate and favorable shape and male advantages like perfect meat quality and strong disease resistance. We obtained $\mathrm{F}_{2}$ population without characteristic segregation from $F_{1}$ self-crossing, which indicated the heterosis in WR could be inherited steadily. Therefore, WR possessed the necessary conditions to form a new genetic variation lineage: (i) nuclear DNA recombination and varia- 


\begin{tabular}{lcccc} 
& \multicolumn{1}{c}{10} & 20 & 30 & 40 \\
RCC-ITS2 & (1) CTCATCGATCGGGGCCTCCGGGTCCCGCGGCTGGAGCTTCGTAGGGGTCG \\
WCC-ITS2 & (1) CTCATCGATCGGGGCCTCCGGGTCCCGCGGCTGGAGCTTCGTAGGGGTCG \\
RW-ITS2 & (1) CTCATCGATCGGGGCCTCCGGGTCCCGCGGCTGGAGCTTCGTAGGGGTCG \\
WR-ITS2 & (1) CTCATCGATCGGGGCCTCCGGGTCCCGCGGCTGGAGCTTCGTAGGGGTCG \\
\hline
\end{tabular}

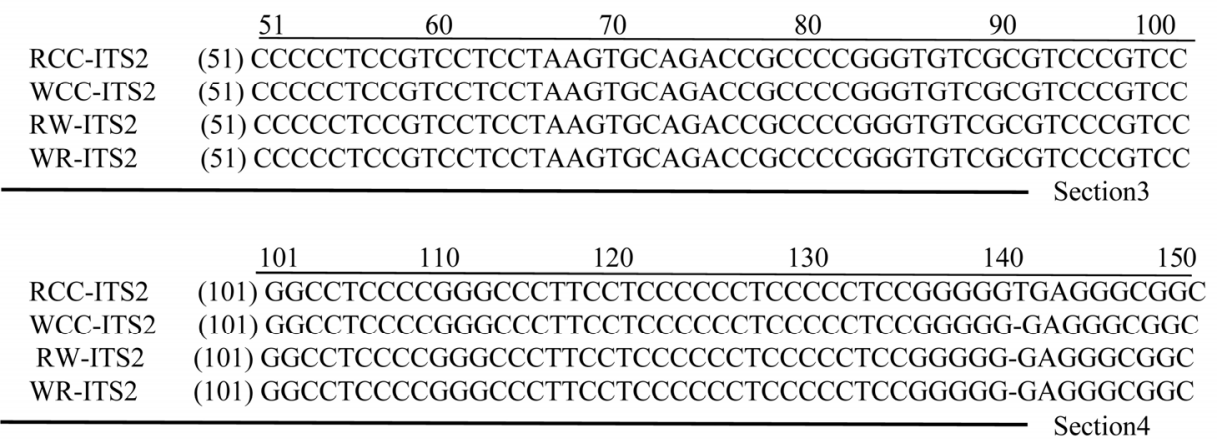

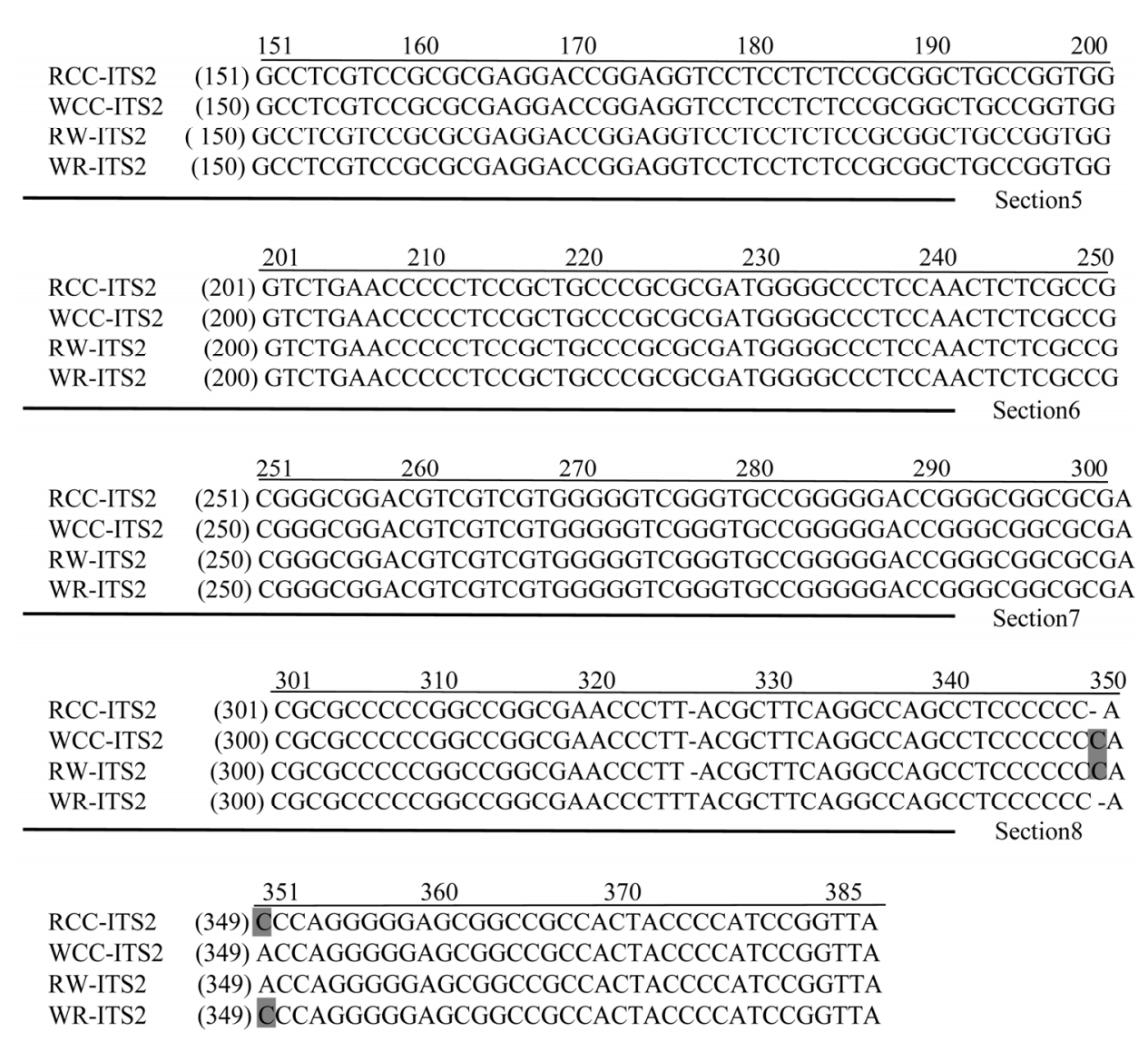

Figure 12 Comparison of the ITS2 sequences from RCC, WCC, RW, and WR.

tion; (ii) effective integration of parent advantages; (iii) bisexual fertile and the heterosis inherited stability. In conclusion, characterization of WR provided new insights into the genotypic variation and phenotypic diversity of fish hybrids obtained from hybridization. In our previous study, both RCC and WCC were commonly used as the female parent to mate with tetraploids in order to produce triploids in aquaculture. The WR gained good characteristics from both
RCC and WCC, bringing a new germplasm to replace WCC and RCC to produce triploid, which will benefit to aquaculture. It is of important significance not only to work on fish breeding, but also to research the genomic variation in hybridization.

The authors declare that they have no conflict of interest. All applicable institutional and national guidelines for the care and use of animals were followed. 
This work was supported by Major International Cooperation Projects of the National Natural Science Foundation of China (31210103918), the National Natural Science Foundation of China (31201980, 31430088 and 31272651), the National High Technology Research and Development Program of China (2011AA100403), the Natural Science Foundation of Hunan Province, China (14JJ3072), Science-Technology Foundation of Hunan Province, China (2014FJ3084), Research Foundation of Education Bureau of Hunan Province, China (13C523), the Cooperative Innovation Center of Engineering and New Products for Developmental Biology of Hunan Province (20134486), the Construct Program of the Key Discipline in Hunan Province, and Construct Program of the National Key Discipline.

1 Bartley DM, Rana K, Immink AJ. The use of inter-specific hybrids in aquaculture and fisheries. Rev Fish Biol Fisher, 2001, 10: 325-337

2 Zhang ZH, Chen J, Li L, Tao M, Zhang C, Qin QB, Xiao J, Liu Y, Liu SJ. Research advances in animal distant hybridization. Sci China Life Sci, 2014, 57: 889-902

3 Liu SJ, Qin QB, Xiao J, Lu WT, Shen JM, Li W, Liu JF, Duan W, Zhang C, Tao M, Zhao RR, Yan JP, Liu Y. The formation of the polyploid hybrids from different subfamily fish crossings and its evolutionary significance. Genetics, 2007, 176: 1023-1034

4 Liu SJ, Liu Y, Zhou GJ, Zhang XJ, Luo C, Feng H, He XX, Zhu GH, Yang $H$. The formation of tetraploid stocks of red crucian carp $\times$ common carp hybrids as an effect of interspecific hybridization. Aquaculture, 2001, 192: 171-186

5 Qin QB, Wang YD, Wang J, Dai J, Xiao J, Hu F, Luo K, Tao M, Zhang C, Liu Y, Liu S. The autotetraploid fish derived from hybridization of carassius auratus red var. (female) $\times$ Megalobrama amblycephala (male). Biol Reprod, 2014, 91: 93

6 Xiao J, Kang X, Xie L, Qin Q, He Z, Hu F, Zhang C, Zhao R, Wang J, Luo K, Liu Y, Liu S. The fertility of the hybrid lineage derived from female Megalobrama amblycephala×male Culter alburnus. Anim Reprod Sci, 2014, 152: 61-70

7 Hu J, Liu SJ, Xiao J, Zhou Y, You CP, He WG, Zhao RR, Song C, Liu Y. Characteristics of diploid and triploid hybrids derived from female Megalobrama amblycephala Yih $\times$ male Xenocypris davidi Bleeke. Aquaculture, 2012, 364-365: 157-164

8 He WG, Qin QB, Liu SJ, Li TL, Wang J, Xiao J, Xie LH, Zhang C, Liu Y. Organization and variation analysis of 5S rDNA in different ploidy-level hybrids of red crucian carp $\times$ topmouth culter. PLoS One, 2012, 7 e38976

9 Wang J, Qin QB, Chen S, Liu SJ, Duan W, Liu JH, Zhang C, Luo KK, Xiao J, Liu Y. Formation and biological characterization of three new types of improved crucian carp. Sci China Ser C-Life Sci, 2008, 51: 544-55

10 Chen S, Wang J, Liu SJ, Qin QB, Xiao J, Duan W, Luo KK, Liu JH, Liu Y. Biological characteristics of an improved triploid crucian carp. Sci China Ser C-Life Sci, 2009, 52: 733-738
11 Luo KK, Xiao J, Liu SJ, Wang J, He WG, Hu J, Qin QB, Zhang C, Tao M, Liu L. Massive production of all-female diploids and triploids in the crucian carp. Int J Biol Sci, 2011, 7: 487-495

12 Liu Y. Propagation Physiology of Main Cultivated Fish in China (in Chinese). Beijing: Agricultural Publishing House, 1993. 22-30

13 Sambrook J, Fritsch EF, Maniatis T. Molecular cloning: a laboratory manual. 2nd ed. Plainsview, NY: Cold Spring Harbor Laboratory Press, 1989

14 Murakami M, Fujitani H. Characterization of repetitive DNA sequences carrying 5SrDNA of the triploid ginbuna ((Japanese silver crucian carp, Carassius auratus langsdorfi). Genes Genet Syst, 1998, 73: 9-20

15 Hall TA. BioEdit: a user-friendly biological sequence alignment editor and analysis program for Windows 95/98/NT. Nucleic Acids Symp Ser, 1999, 41: 95-98

16 Thompson JD, Higgins DG, Gibson TJ. CLUSTALW: improving the sensitivity of progressive multiple sequence alignment through sequence weighting, position-specific gap penalties and weight matrix choice. Nucleic Acids Res, 1994, 22: 4673-4680

17 Barton NH. The role of hybridization in evolution. Mol Ecol, 2001, 10: $551-568$

18 Mallet J. Hybrid speciation. Nature, 2007, 446: 279-283

19 Seehausen O. Hybridization and adaptive radiation. Trends Ecol Evol, 2004, 19: 198-207

20 Liu SJ. Distant hybridization leads to different ploidy fishes. Sci China Life Sci, 2010, 53: 416-425

21 Stelkens R, Seehausen O. Genetic distance between species predicts novel trait expression in their hybrids. Evolution, 2009, 63: 884-897

22 Li L, Zhong ZZ, Zeng M, Liu SJ, Zhou Y, Xiao J, Wang J, Liu Y. Comparative analysis of intermuscular bones in fish of different ploidies. Sci China Life Sci, 2013, 56: 341-350

23 Liu SJ. Fish Distant Hybridization (in Chinese). Beijing: Science Press, 2014. 6-18

24 Murakami M, Matsuba C, Fujitani H. The maternal origins of the triploid ginbtma (Carassius auratus langsdorfi): phylogenetic relationships within the $C$. auratus taxa by partial mitochondrial D-loop sequencing. Genes Genet Syst, 2001, 76: 25-32

25 Luo J, Gao Y, Ma W, Bi XY, Wang SY, Wang J, Wang YQ, Chai J, Du R, Wu SF, Meyer A, Zan RG, Xiao H, Murphy RW, Zhang YP. Tempo and mode of recurrent polyploidization in the Carassius auratus species complex (Cypriniformes, Cyprinidae). Heredity, 2014, 112: 415-427

26 You CP, Zhao RR, Hu J, Liu SJ, Tao M, Zhang C, ChenYB, Qin QB, Xiao J, Duan W, Liu Y. Inheritance of the complete mitochondrial genomes in three different ploidy fishes. Curr Mol Med, 2014, 14: $1322-1330$

27 Allen SK, Wattendorf RJ. Triploid grass carp: status and management implications. Fisheries, 1987, 12: 20-24

Open Access This article is distributed under the terms of the Creative Commons Attribution License which permits any use, distribution, and reproduction in any medium, provided the original author(s) and source are credited. 